Supporting information

\title{
Guanidinium Chloride Passivated Perovskites for Efficient Solar Cells: The Role of Passivating Solvent
}

Mingguang Li, Ying Zhang, Huan Gao, Ying Peng, Senlin Tang, Longsheng Yu, Runfeng

\author{
Chen* and Wei Huang
}

Key Laboratory for Organic Electronics and Information Displays \& Jiangsu Key Laboratory for

Biosensors, Institute of Advanced Materials (IAM), Jiangsu National Synergetic Innovation Center for Advanced Materials (SICAM), Nanjing University of Posts \& Telecommunications, 9 Wenyuan Road, Nanjing 210023, China.

(a) $\mathrm{Pbl}_{2}$

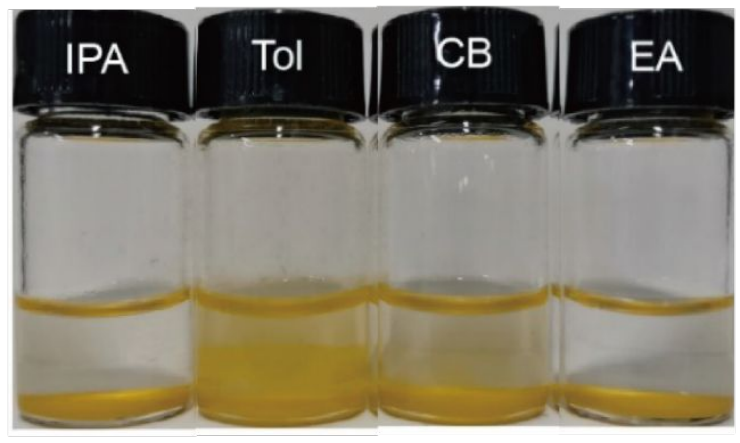

(b) MAI

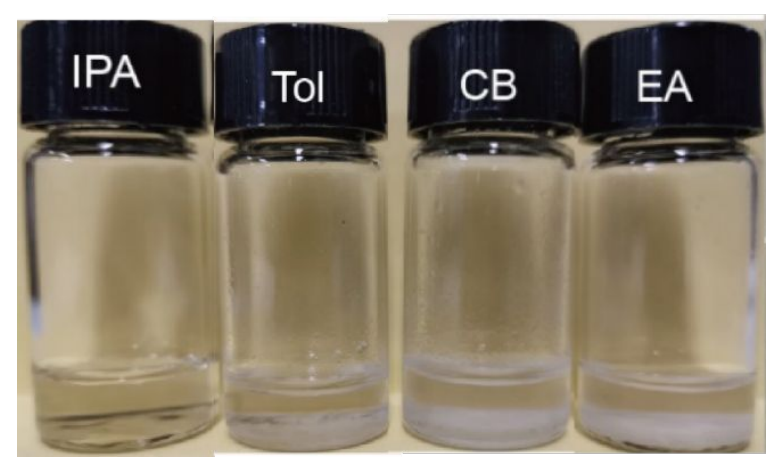

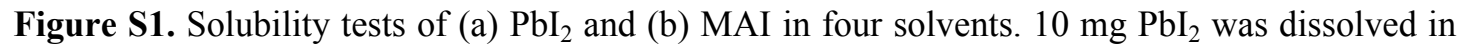
four solvents $(2 \mathrm{~mL}) .20 \mathrm{mg}$ MAI were dissolved in four solvents $(1 \mathrm{~mL})$. 


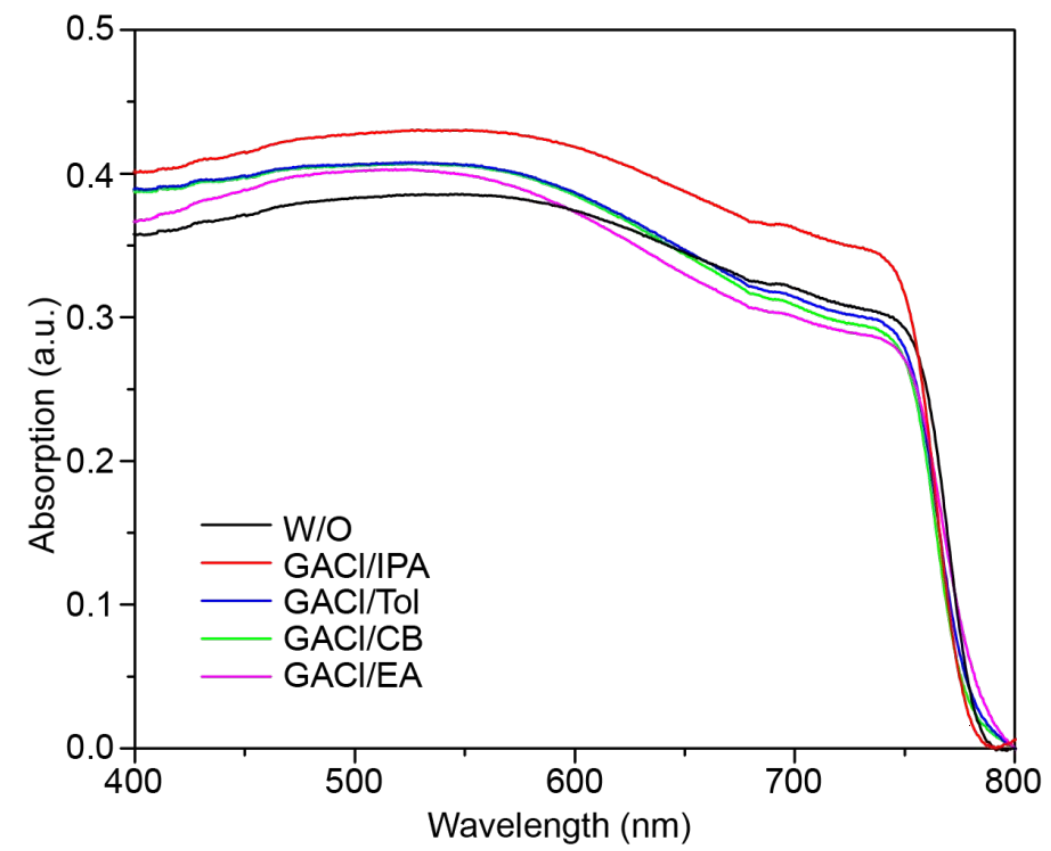

Figure S2. UV-vis absorption spectra of $\mathrm{MAPbI}_{3}$ films prepared with and w/o GACl passivating treatment.

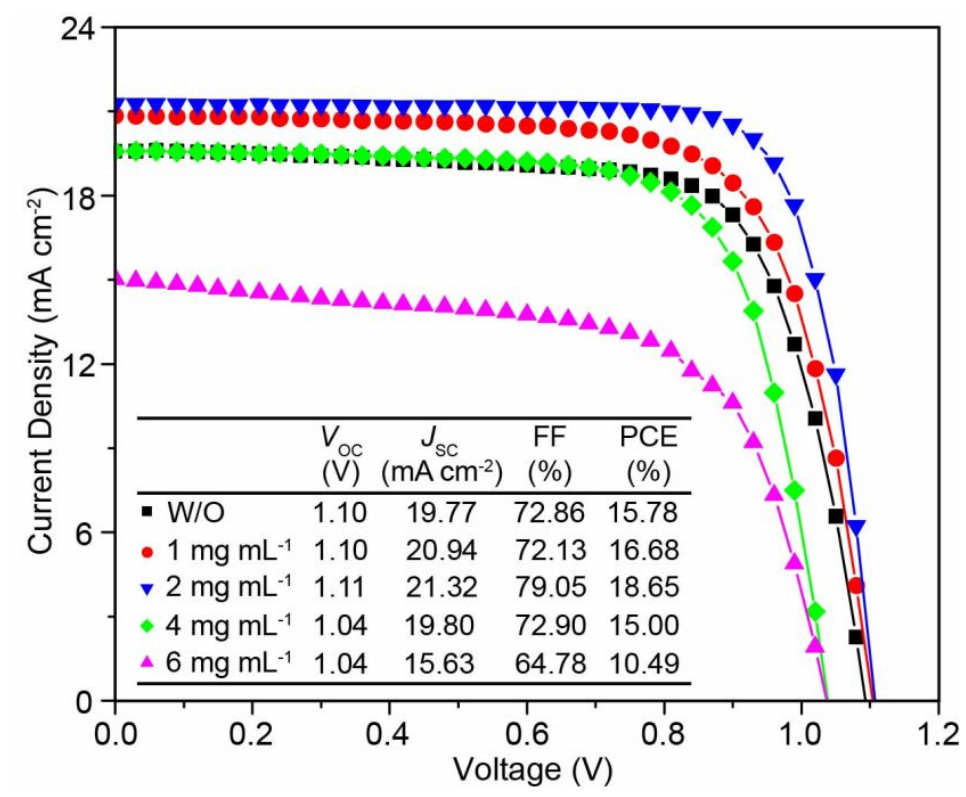

Figure S3. Current density-voltage $(J-V)$ curves for inverted PSCs with different concentration treatments of $\mathrm{GACl}$ under illumination of an AM 1.5 solar simulator in air. 


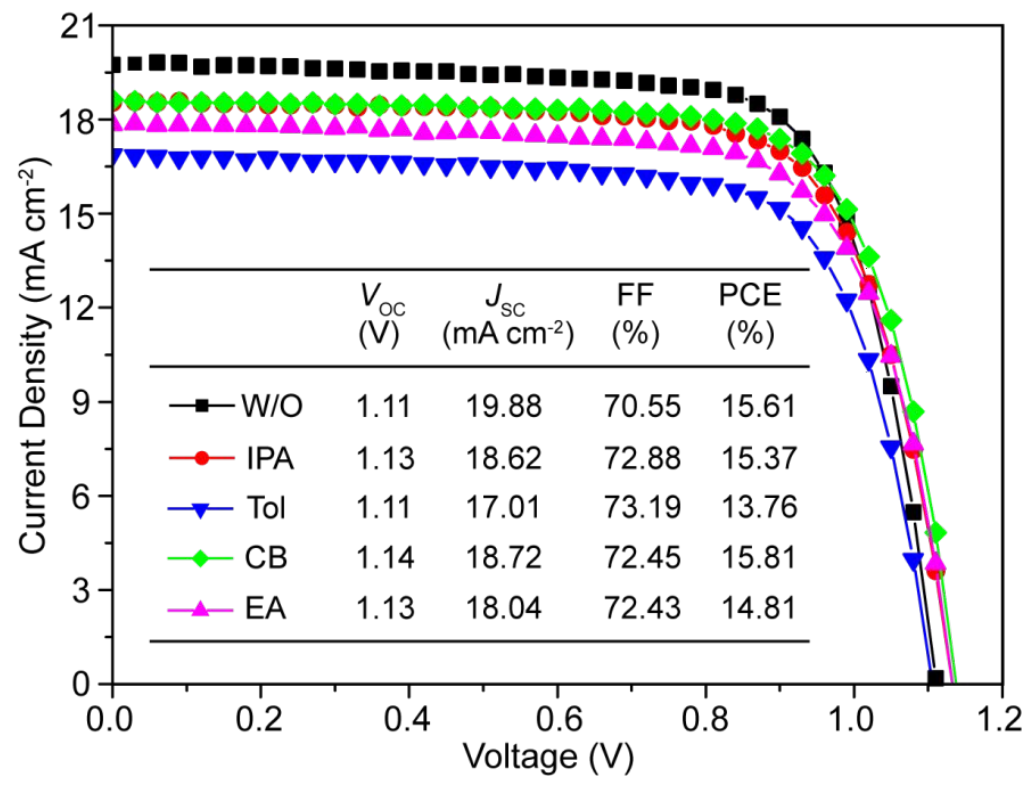

Figure S4. Current density-voltage $(J-V)$ curves for inverted PSCs with different solvent treatments under illumination of an AM 1.5 solar simulator in air. 

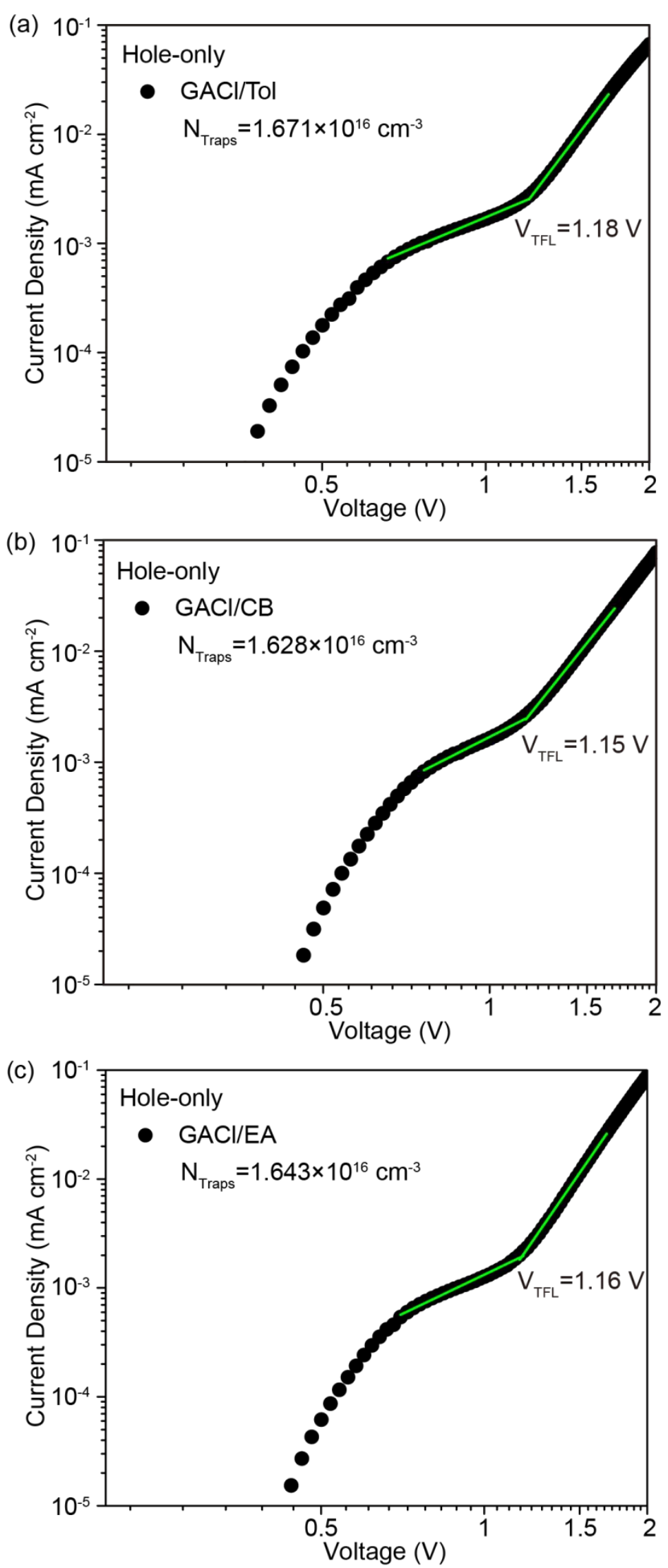

Figure S5. $J-V$ characteristics of the hole-only devices measured in dark. (a) GACl/Tol passivated device, (b) GACl/CB passivated device, and (c) GACl/EA passivated device. 


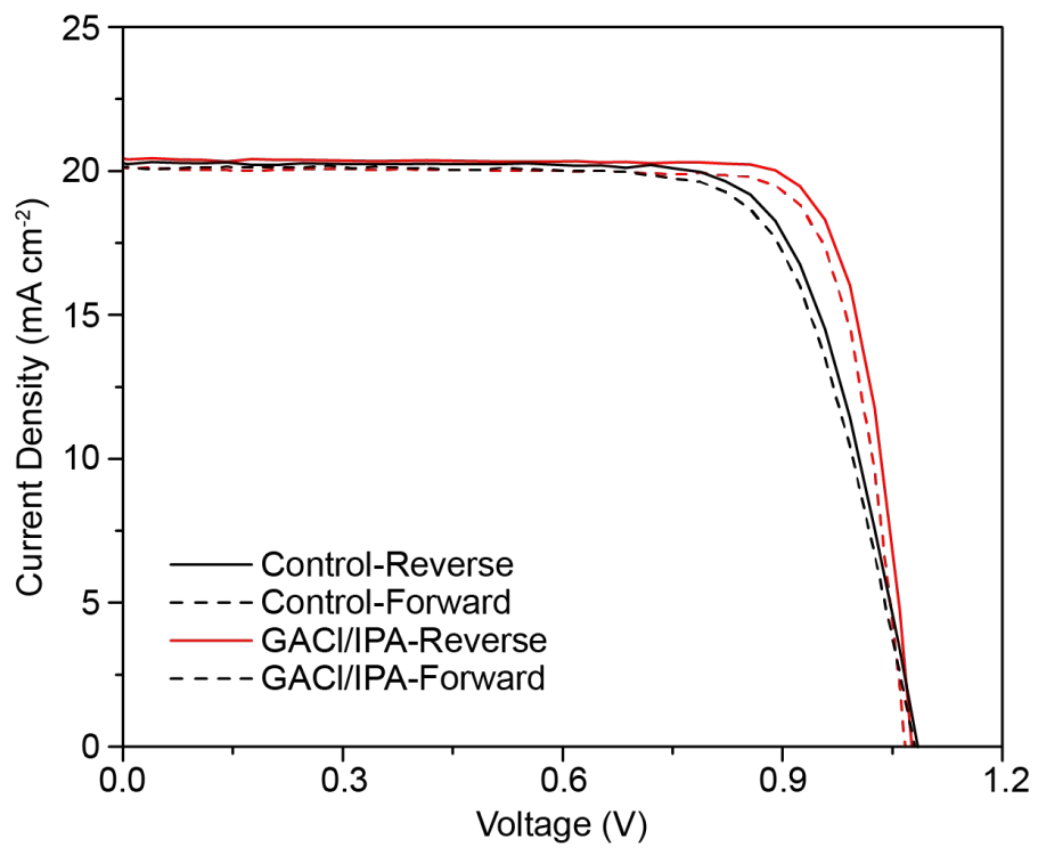

Figure S6. $J-V$ hysteresis curves of perovskite solar cells with and w/o GACl/IPA treatment. 